\title{
Sur les collectifs humains
}

\author{
Jihene Boughanmi \\ EA 1339 LiLPa (Linguistique, Langue, Parole), Université de Strasbourg, France.
}

\begin{abstract}
Résumé. Les noms collectifs constituent une classe hétérogène regroupant des entités animées et inanimées. Dans le cadre de cette contribution nous nous intéresserons aux noms collectifs humains. L'approche adoptée vise à présenter des généralités ainsi que des particularités de cette classe de noms. Des généralités à travers la présentation d'une typologie. Et des particularités à travers l'examen d'un cas de collectif humain : peuple.
\end{abstract}

\begin{abstract}
About collective human nouns. Several types of referential entities are classified under the label collective : entities that refer to inanimates as well as entities that refer to animates. In this contribution, we will be focusing on collective human nouns. Our approach aims at presenting the general characteristics as well as the distinctive features of this class of nouns. The general characteristics through the presentation of a typology and their peculiartities through the study of a human collective noun in particular : people.
\end{abstract}

\section{Introduction}

Plusieurs types d'entités référentielles sont rassemblés sous l'étiquette du collectif; celles qui renvoient aux inanimés ainsi que celles qui renvoient aux animés; animaux et humains. Dans cette contribution nous nous intéresserons aux noms collectifs humains, qui dans l'état actuel sont peu étudiés ${ }^{1}$. Nous commencerons par présenter cette classe de noms pour ensuite proposer une typologie. Enfin, nous terminerons par l'étude d'un nom collectif humain : peuple, pour rendre compte de quelques spécificités.

\section{Du nom collectif au nom collectif humain}

\subsection{Retour sur la notion du collectif}

Sur le plan pragmatique, il est aisé d'admettre que « bouquet» contrairement à « fleur », présente l'idée d'une pluralité interne. Dans le cadre de ce travail, nous ne passerons pas en revue les travaux sur les noms collectifs, nous nous baserons uniquement sur ceux de Lammert (2010), qui, dans son étude sur les noms collectifs (désormais NC), définit cette classe à partir de leurs principales caractéristiques, à savoir :

\footnotetext{
i. une discordance morpho-sémantique entre la pluralité interne que ces noms véhiculent et l'absence d'une quelconque marque de pluriel morphologique ;

ii. une certaine homogénéité interne: les éléments de la collection que représente le Ncoll sont d'une même catégorie ou du même type ;

iii. une hétérogénéité existant entre élément et collection: la nature des éléments est différente de celle de la collection, et le lien qui les unit peut être assimilé à une relation partie-tout. (Lammert, 2010: 69)
}

Nous adopterons cette approche comme cadre général de notre objet de travail. 


\subsection{Quant le trait [+hum] s'ajoute au collectif}

Un nom collectif humain (désormais $\mathrm{NCH}$ ) a, en outre, la faculté de désigner exclusivement des collectivités humaines. En d'autres termes c'est un nom « dont une des acceptions, stabilisée ou en cours de stabilisation, dénomme un rassemblement d'humains conçu sous un certain angle » (Lecolle, 2014). En observant les définitions lexicographiques ${ }^{2}$ de notre classe de noms récoltées à partir du $T L F i$, cette référence à l'humain s'opère sur quatre niveaux :

- des noms dont la principale acception renvoie à l'humain

Peuple : ensemble des humains vivant en société sur un territoire déterminé et qui, ayant parfois une communauté d'origine présentent une homogénéité relative de civilisation et sont liés par un certain nombre de coutumes et d'institutions communes. Famille : ensemble constitué par un couple de parents et leurs enfants.

- des noms qui, par métonymie, désignent l'humain

Banque : sens1: Service public ou privé se chargeant des opérations de dépôt, d'achat, de prêt, de vente, auxquelles peut donner lieu le commerce de l'argent et des titres négociables.

Sens2 : Ensemble des personnes exerçant une fonction de direction dans les banques.

- des $\mathrm{NCH}$ généraux ${ }^{3}$ qui renvoient à l'humain

Humanité : ensemble des hommes, du genre humain, parfois considéré comme un tout, un être collectif.

Biotype : [biologie] groupe d'individus possédant le même génotype (...), groupe d'individus ayant le même patrimoine héréditaire, homozygote.

: [génétique] Type d'individus présentant les mêmes dominantes biopsychologiques (déterminations embryologiques, caractéristiques morpho-physiopsychologiques).

- des NC généraux ${ }^{4}$, qui peuvent désigner l'humain

Groupe : Ensemble de personnes ayant des caractéristiques communes.

Classe : Ensemble d'êtres réunis en raison des traits qui leur sont communs.

Cette structuration sur quatre niveaux nous offre un panel assez large de $\mathrm{NCH}$.

\section{Vers une typologie des $\mathrm{NCH}$}

Un examen rapide des paraphrases utilisées dans le TLFi pour définir les NCH fournit quelques éléments de typologie. Nous trouvons des indications sur la taille des groupes : «ensemble de deux personnes », «ensemble de trois personnes », «petit groupe de », sur le mode de regroupement : «rassemblement de personnes », « assemblée de personnes », « classe sociale », ainsi que des définisseurs génériques du type «groupe de personnes », «groupe d'individus » ${ }^{5}$, « ensemble de tous ceux qui », etc.

Les configurations des relations humaines étant très hétérogènes, nous nous confrontons au problème de la dénomination des groupes humains. Avant d'établir une typologie, il convient de fournir des indications sur la structure de la collection elle-même.

\subsection{Des touts hiérarchiques vs des touts non hiérarchiques}

Comme le souligne Flaux «les individus collectifs humains peuvent présenter une structure interne hiérarchisée » (1999: 479). Cette hiérarchisation concerne des noms comme armée, famille, etc. où l'ensemble est formé soit d'éléments de statuts différents (1), soit de sous-ensembles différents (2). Ainsi, 
1) Le directeur général de la commission des terres a fait diffuser une circulaire expliquant qu'une «famille » se compose d'un mari, sa femme et leurs enfants. (Réponses du gouvernement Soudanais à la liste des points à traiter $(\mathrm{CCPR} / \mathrm{C} / \mathrm{SDN} / \mathrm{Q} 3)$ à l'occasion de l'examen du troisième rapport périodique su Soudan, Comité des droits de l'homme, $2007: 6$ ).

2) Dans sa composante militaire, l'armée de terre est composée de trois grandes catégories. Tout d'abord, les militaires du rang. Ils sont plus de 60000 et sont tous des contractuels. Les sous-officiers sont environ 42000 et les officiers de l'ordre 16000. (Google, www.recrutement.terre.defense.gouv.fr ).

Ces statuts peuvent être plus ou moins saillants. Dans l'exemple suivant, l'énoncé fait abstraction de cette structuration en considérant les membres de la famille comme des personnes :

3) Que dois-je faire si ma famille est composée de plus de six personnes ? (Google).

Cette hiérarchisation peut générer une ambiguïté dans l'interprétation de quelques énoncés. Dans (4), il est difficile de savoir si c'est les parents ou tous les membres de la famille qui ont décidé de déménager.

4) Ma famille a décidé de déménager.

Borillo soulève le même problème dans le cas des noms d'organisations utilisés en tant que noms collectifs :

- La SNCF (employés ?) fait grève lundi prochain. La SNCF (membres dirigeants ?) a pris des mesures de redressement financier. (Borillo, $1997: 117)$

A l'inverse, se présente un autre type de structure où les éléments sont «similaires » et sont au même niveau. La bourgeoisie est formée de bourgeois, le public de spectateurs, etc.

\subsection{Des regroupements par défaut vs des regroupements construits}

Cette distinction opère sur le mode de formation de l'ensemble. Il existe des regroupements dans lesquels l'être humain se retrouve par défaut. Cela concerne les noms marqués d'un point de vue identitaire ; race, ethnie, peuple, nation, etc., les noms de classes sociales ; bourgeoisie, noblesse... ou encore les noms désignants des liens sociaux, famille, fratrie, parenté, etc. D'où l'impossibilité des énoncés suivants :
5) *J'ai rejoint une ethnie
6) *J'ai créé la bourgeoisie
7) *J'ai supprimé une race

Les regroupements construits sont formés par l'homme. Ils sont susceptibles, à un certain degré, de manipulations, nous pouvons les former, fonder, constituer, rejoindre, supprimer, remplacer, etc.

8) Pedro Miguel Pauleta verrait bien Cristiano Ronaldo rejoindre le Paris-SG. (Google).

9) Ainsi, le conseil peut créer un comité pour l'aider à résoudre une question importante ou un problème particulier - comme la politique du conseil en matière de conflits d'intérêts ou la politique relative à l'adhésion à l'organisation. (Google).

10) Les membres élus FO n'accepteront pas de voir aux portes alimentaires restantes, des salariés extérieurs à l'Entreprise, et vous demandent de prendre des décisions concernant ces sociétés, avant même de supprimer le personnel Auchan. (Google). 


\subsection{Une tentative de typologie}

Après avoir apporté ces indications sur la structuration des ensembles, nous tenterons dans ce qui suit d'établir une typologie provisoire fondée sur les modes de regroupements :

- regroupement spatial: ces groupes sont constitués d'éléments partageants le même espace spatial $^{6}$ (une place, une rue, etc.). Ils peuvent être statiques ou en mouvement. Cette configuration ne concerne pas la finalité du rassemblement mais s'intéresse au rassemblement lui-même.

- $\quad$ regroupement social : il repose sur la répartition des personnes à l'intérieur de la société. Cela concerne une position stratifiée relative à une certaine classe sociale, ou les liens relationnels sociaux (fratrie, voisinage, etc.). Les strates sociales peuvent aussi qualifier, d'un point de vue axiologique, les individus en les considérant soit positivement (élite, crème, etc.) soit négativement (populace, racaille, etc.).

- regroupement fonctionnel : les groupes humains sont aussi construits selon une finalité, ou une fonction communes qui les unissent. Les membres peuvent être plus ou moins impliqués. C'est ce qui oppose par exemple clientèle à jury. Par définition, la clientèle est « un ensemble de personnes qui achètent régulièrement des services ou des choses dans un établissement commercial » $(T L F i)$. Cette habitude d'achat, individuelle au départ ${ }^{7}$, multipliée par un certain nombre de personnes forme un collectif humain, poursuivant un même but. Les membres d'un jury qui constituent «une commission temporaire, occasionnelle ou exceptionnelle chargée officiellement de l'examen d'une question » (TLFi), ont une fonction commune et œuvrent tous à l'accomplissement d'une tâche précise. De ce fait, les finalités peuvent varier du moins fort (clientèle) au plus fort (jury).

Le tableau (1) ci-dessous illustre cette typologie avec des exemples pour chaque sous classement.

\begin{tabular}{lll}
\hline Classement & Sous classement & Exemples \\
\hline Spatial & Statique & Foule, rassemblement, public, etc. \\
& Déplacement/mouvement & Cortège, marche, cordée, etc. \\
\hline Social & Strate & Peuple, bourgeoisie, aristocratie, etc. \\
& Lien social & Famille, voisinage, fratrie, etc. \\
& Axiologique & Racaille, élite, canaille, crème, etc. \\
\hline Fonctionnel & - & clientèle, lectorat, etc. \\
& & \\
& & \\
& & commission, jury, etc.
\end{tabular}

Tableau 1. Classification sémantique des $\mathrm{NCH}$ 


\section{Etude de cas : peuple}

Pour rendre compte de quelques spécificités concernant les $\mathrm{NCH}$, nous avons choisi d'étudier le nom «peuple». Ce choix nous semble pertinent dans la mesure où ce substantif, en raison de sa polysémie intègre plusieurs catégories possibles de $\mathrm{NCH}$.

Pour analyser le fonctionnement de peuple, nous avons eu recours aux bases de données Frantext et Wortschatz $^{8}$. A partir de Frantext, nous avons établi un corpus regroupant des occurrences de peuple, sur la période de 2000 à 2010 dans tous les genres textuels exclusion faite du théâtre et de la poésie ${ }^{9}$. Au-delà du corpus, Wortschatz ${ }^{10}$ fournit de plus amples informations sur l'environnement syntagmatique de peuple.

\subsection{Un nom polysémique}

Pour rendre compte des différentes configurations possibles dans la dénomination de peuple, nous avons choisi de décrire tout d'abord, les sens par les traits sémantiques suivants en nous appuyant sur les définitions du TLFi :

- $\quad$ [+espace] : pour les ensembles qui partagent un espace commun, un territoire bien défini.

- $\quad$-espace] : pour l'ensemble de personnes qui ont une existence en dehors d'un même espace.

- $\quad[+$ fonctionnel] : les éléments de la collection, qu'est le peuple, sont réunis du fait de propriétés partagées, de fonctions communes.

- [+social] : pour l'emploi de peuple en tant que classe sociale.

L'articulation de ces traits fournit à chaque fois un sens différent :

$\checkmark \quad$ [+espace] + [+fonctionnel] : le peuple nation : «ensemble des humains vivants en société sur un territoire déterminé, et qui ayant parfois une communauté d'origine, présentent une homogénéité relative de civilisation et sont liés par un certain nombre de coutumes et d'institutions » (TLFi)

11) Il parle du peuple de France à la manière de Louis XVI, et n'y a plus à se tromper sur les gaullistes. (M. Duras, Cahiers de la guerre et autres textes, 2006, p 233).

12) Les grecs sont heureux et fiers, et leur roi envoie des messages tordants : «Je suis fier de régner sur un tel peuple ». (D. Domenach-Lallich, Journal d'une adolescente (novembre 1939-1944), 2001, p 61).

$\checkmark \quad$ [-espace] + [+fonctionnel] : pour les collectivités ethniques, raciales et religieuses : le peuple sioux, le peuple noir, le peuple chrétien ...

13) A cette époque, chacun conservait en tête les propos atterrants de de Gaulle sur le peuple juif, d'autant plus injuste que l'offensive de d'Israël en 1967 relevait d'une sorte de légitime défense. (S. Veil, Une vie, 2007, p 165).

$\checkmark \quad$ [+fonctionnel] + [+social] : l'emploi de peuple comme classe sociale.

14) L'accord honteux fut en effet signé, ce qui, sur le moment, fit le bonheur de la plupart des Européens, qu'ils soient ignares ou cultivés, qu'ils appartiennent aux peuple ou à l'élite. (M. Storti, L'arrivée de mon père en France, 2008, p 90).

$\checkmark \quad$ [+espace] : pour les populations d'agglomérations, de villes, etc.

15) Après un bain merveilleux, je me couchai, et ne pus dormir à cause des moustiques d'une part, et, d'autre part, des rumeurs de ce peuple napolitain qui ne connaît pas le sommeil nocturne. (M. Havet, Journal 1919-1924, 2005, p 451). 
: pour l'acception massive de peuple, un emploi synonyme de foule.

16) Il y a du peuple dans la rue.

Cette configuration pour peuple montre que les répartitions «fonctionnel», «spatial» et «social» peuvent se combiner entre elles ${ }^{11}$. Dans l'acception de peuple «nation» Le trait [+spatial] vient compléter le trait [+fonctionnel].

\subsection{Peuple : singulier morphologique et pluriel sémantique}

L'une des caractéristiques propres aux noms collectifs concerne l'opposition entre leur forme morphologique et leur référence ontologique, ce qui leur confère un statut intermédiaire les situant entre l'unicité et la pluralité.

Peuple, renvoie à un ensemble d'humains sur le plan lexical et sémantique. Pourtant, aucun morphème de pluriel ne traduit cette pluralité, démontrable pourtant syntaxiquement à l'aide des tests habituels ${ }^{12}$. Ainsi, il est compatible avec les prédicats verbaux à arguments pluriels à savoir ; les verbes de rassemblement, comme dans (17), les verbes dénotant le grand nombre (18) et les verbes de dispersion (19), qui utilisés avec la préposition en, comme dans l'exemple (20), font «éclater le référent collectif en obtenant différentes sous-collections ou en donnant les différentes entités qui la composent » ( Dubois et DuboisCharlier, 1996 et Guillet, 1978 : 213-214, in Lammert, 2010).

17) En 1989, quand le peuple Allemand s'est retrouvé et s'est réuni, un parrainage s'est instauré entre Hude et Kröpelin, une ville située au nord-est de l'Allemagne. (Compte rendu du conseil municipal du mercredi 3 février 2016, Ville d'Arnage).

18) Le peuple grouille dans la rue. (A. Montoux, DicoJOB 2010 Concepts et vocabulaire des organisations, 2010, 618).

19) Si l'on ne descend pas vers lui, le peuple se sépare et se disperse. (C. Le Blanc, Le Wen Zi : à la lumière de l'histoire et de l'archéologie, 2000).

20) Dites aux citoyens: Enrichissez-vous! et le peuple éclate en autant d'individualités concurrentes. (Google).

Peuple est également compatible avec les adjectifs, adverbes et prépositions qui permettent de rendre compte de la pluralité interne des noms collectifs, comme le prouve les exemples suivants :

21) Sur la plage blanche surgissaient les masques de sa scène intérieure, un peuple nombreux, bariolé(...) (G. Aubry, Personne, 2009, p 13).

22) Le peuple, unanime, s'est prononcé contre cette injustice.

23) Que sert, après cela, de dire que les peuples seront plus disposés de séparer leur cause de celle des rois; que l'on ne corrompt pas le peuple en masse. (Le pour et le contre, 1792)

Pour le test de la reprise anaphorique, dans le cas des noms [+animés], Lammert utilise l'anaphore indirecte collective exprimée par le pronom «ils », qui reprend les éléments de la collection.

24) A l'hôpital, ils l'ont bien soigné. (Lammert, $2010: 90$ ).

Or, dans le cas de notre nom et dans tout le corpus, la reprise anaphorique est assurée par le pronom singulier « il », où peuple est présenté comme une seule unité.

25) Temps de l'analyse où ça le peuple ne veut plus se faire représenter. Il veut le pouvoir directement, sans médiateur, il récuse les délégués. Il est tyrannique. (J. Bertrand, Représentants du peuple, 2000, 130-132).

Cependant, il existe des cas de figures où peuple est repris par des adjectifs de nationalité en emploi nominal comme les français, les allemands...etc. 


\subsection{Homogénéité interne ?}

Nous avons vu, avec les tests mis en avant précédemment, que le nom peuple véhicule sémantiquement l'idée d'une pluralité. A ce même niveau interne se manifeste, également, une homogénéité caractéristique des noms collectifs qui suppose que : « les éléments qui forment la collection sont de même nature, qu'ils sont identiques, qu'ils appartiennent à la même catégorie » (Lammert, 2010, 102).

Lammert considère que le principe de l'homogénéité est applicable à la fois aux noms massifs ainsi qu'aux noms collectifs. Toutefois, l'homogénéité des noms collectifs est seulement interne, c'est-à-dire que les éléments sont identiques les uns par rapport aux autres, et non par rapport au tout qu'ils constituent, comme c'est le cas pour les massifs. Les éléments d'une collection pour un nom collectif ont une existence autonome en dehors du tout qu'ils représentent. Dans le cas de peuple, personne, si on peut le considérer comme l'élément constitutif de l'ensemble peuple, n'appartient pas systématiquement à peuple, il possède son propre sémantisme et est considéré comme «individu de l'espèce humaine, sans distinction de sexe. » (TLFi).

L'analogie avec les noms massifs ne s'arrête pas ici. En effet, les noms collectifs et les massifs partagent les principes de « référence cumulative » et de « divisibilité », révélateurs de l'homogénéité interne.

Lammert applique le principe de la référence cumulative ou de «l'addition » aux noms collectifs et ce, en suivant le modèle des noms massifs appliqué au pluriel, car " si l'on a un ensemble formé par une pluralité d'individus atomiques à laquelle on rajoute de ces mêmes individus, le principe de la référence cumulative est respecté » (Lammert, 2010, 105). Ainsi :

26) Des hommes+des hommes=des hommes

27) Un troupeau+un troupeau=un troupeau plus grand (si l'on considère que les deux troupeaux sont constitués d'éléments identiques)

28) Un peuple+un peuple $=$ deux peuples

Dans (28), le résultat obtenu avec la référence cumulative ne donne pas une entité homogène. Peuple obéit plutôt au principe de "référence cumulative hétérogène ». Les éléments qui définissent un peuple sont considérés en fonction de plusieurs liens qui peuvent les unir, et quel que soit le lien, le tout obtenu est unique. Ainsi, l'addition du peuple français au peuple chinois donne deux peuples bien différents.

Le principe de la divisibilité se définit par le fait que si nous ôtons quelques éléments d'une collection, ce qui en reste forme toujours une collection de la même nature : " la soustraction d'un membre n'ôte rien à l'identité de la collection, jusqu'à un certain seuil quantitatif »(Lecolle, 1998, 51).

Or, considérant le nom peuple, par référence au peuple français, nous pouvons difficilement affirmer que si nous enlevons les Français de Paris, le reste obtenu formera toujours le peuple français ${ }^{13}$.

En effet, peuple, dans presque tous les sens auxquels il renvoie, désigne des ensembles considérés dans la totalité. D'ailleurs, dans les différentes définitions des dictionnaires, peuple est introduit par « l'ensemble de », ce qui donne à entendre que l'ensemble qu'il vise comprend la totalité des individus, considérés à chaque fois en fonction d'un critère bien déterminé. Le principe de la divisibilité ne peut alors s'appliquer à peuple. Il existe cependant, une exception : dans le cas où peuple est synonyme de foule et désigne simplement une multitude de personnes rassemblées, nous pouvons soustraire quelques éléments de la collection sans toucher à sa référence ${ }^{14}$.

29) A la nuit tout un peuple se massait devant la pagode (Le Petit Robert). 


\subsection{Peuple et ses éléments : une relation hétérogène}

\subsubsection{Une relation du type partie-tout}

La dernière caractéristique des noms collectifs correspond à l'hétérogénéité « ontologique » et « référentielle ${ }^{15}$ qui existe entre la collection et les éléments qui la constituent. En effet, la collection n'est pas de même nature que les éléments qui la composent. Ensemble, ils entretiennent une relation du type partie-tout. Pour illustrer ce type de lien dans le cas de peuple, nous nous baserons sur les travaux de Cruse (1986).

Dans une optique méronimique qui désigne « the semantic relation between a lexical item denoting a part and that denoting the corresponding whole » (D. Cruse, 1986, 159), Cruse considère les collections selon trois types de relations :

- Relation group/member :

Cette relation méronimique regroupe les entités, ayant le trait [+humain], tel que famille, jury, orchestre, etc. en groupes qui possèdent un but ou une fonction en commun : « they designate groupings which have a purpose or function » (D. Cruse, 1986, 176).

- Relation class/member

Les regroupements d'humains peuvent aussi former des «classes » dont la réunion est justifiée par la possession d'attributs en commun : « a class is an assemblage of humans justified more by the possession of common attributes than a common purpose » (D. Cruse, 1986, 176). C'est ce type de relation qui unit le prolétariat et un ouvrier, l'aristocratie et un duc, ou encore le clergé et un évêque.

- Relation collection/member

Cette relation concerne typiquement les inanimés et qualifie les rapports que peuvent entretenir par exemple : arbre et forêt, bouquet et fleur...

Comme nous l'avons vu précédemment, peuple est un nom polysémique. Le terme de polysémie est simplement utilisé, ici, pour désigner la multiplicité de sens associée à une unité lexicale. De ce fait, peuple est compatible avec les trois entités collectives de Cruse selon son sens.

Ainsi, il peut former une entité du type « groupe »; ce type concerne les emplois où peuple est considéré, d'un point de vue fonctionnel, comme un ensemble de personnes ayant un but ou une fonction en commun. Nous retrouvons souvent avec ce type d'emploi des expansions qui spécifient ces groupes : des adjectifs de nationalité (30), des adjectifs qui renseignent sur l'ethnie (31), sur l'activité professionnelle (32), etc. :

30) Perceptif plus qu'on le pense, le peuple français y avait trouvé un champ pourvoyeur d'héroïsme, l'avait fait sien en le transformant en hymne de la Résistance et de la liberté intemporelle. (Y. Szczupak-Thomas, Un diamant brut, 2008, 341-342).

31) À la pointe orientale de l'île, Santiago de Cuba, un peuple noir, c'est l'âme afro-cubaine. (D. Perrut, Partia o muerte, 2009, 167-168).

32) Comment admettre qu'un peuple essentiellement commerçant, qui avait fait preuve d'une passivité fatale face à l'entreprise nazie, se change du jour au lendemain en héros porteurs des vertus. (Y. Szczupak-Thomas, Un diamant brut, 2008, 409-410).

Comme le prolétariat ou l'aristocratie, peuple, quand il désigne un ensemble de personnes qui n'appartiennent pas aux classes dominantes ou qui sont caractérisées par la vulgarité, forme une entité référentielle du type « classe »:

33) Ces études reposent sur les préjugés d'instituteurs humanistes qui persistent à opposer une élite détentrice des vraies valeurs (eux-mêmes) au peuple qu'il s'agit d'amener à la culture. (G. Perec, Entretiens et conférences I (1965-1978), 2003, 97-98). 
34) 54) Nous savons que nous ne sommes pas du peuple, et elle comme nous, si par moments cette position élevée nous donne quelque fierté, c'est plutôt de la séparation d'entre notre classe et ce peuple que nous tenons le tourment principal de notre vie. (P. Guyotat, Formation, 2007, 137-138).

D'un point de vue spatial, peuple, en tant que substantif quantificateur ${ }^{16}$ peut être considéré comme un ensemble du type collection dont les membres sont explicités par des syntagmes binominaux. Cela concerne le cas où peuple réfère aux inanimés :

35) En un mot, les statues de prix étaient si nombreuses, qu'il fallut créer des officiers pour garder nuit et jour ce peuple de statues, et ces troupeaux de chevaux, si je puis parler ainsi, dispersés dans toutes les rues palais et places publiques de la ville. (M. De Felice, Encyclopédie, ou dictionnaire universel raisonné des connaissances humaines, volume 6 , 1770, 176)

\subsubsection{Quel méronyme pour peuple?}

A la différence d'un nom comme «feuillage » où la récupération du nom d'élément (feuille) est assez évidente morphologiquement, peuple comme certains autres $\mathrm{NCH}^{17}$, ne dispose pas de méronyme stable. Pour faire ressortir une relation méronomique entre deux unités lexicales $\mathrm{X}$ et $\mathrm{Y}$, et pour monter que $\mathrm{X}$ est le méronyme de Y, Cruse (1986) utilise les paraphrases : un X est une partie de Y, et un Y a un ou des X. Celles-ci s'appliquent aux noms considérés d'une manière générale : « $\mathrm{X}$ is a meronym of $\mathrm{Y}$ if and only if sentences of the for a $\mathrm{Y}$ has Xs/an $\mathrm{X}$ and an $\mathrm{X}$ is a part of $\mathrm{Y}$ are normal when the nouns phrases an $\mathrm{X}$, a Y are interpreted geerically ». (Cruse, 1986, 160). Or, dans le cas de peuple, les paraphrases par les relateurs «avoir » ou « être une partie de » se révèlent inadéquats, compte tenu de leurs sémantisme :

$$
\begin{aligned}
& \text { 36) *Paul est une partie du peuple } \\
& \text { 37) *Le peuple a Paul }
\end{aligned}
$$

Cependant, pour mieux décrire la relation de peuple avec ses potentiels composants, nous pouvons avoir recours à d'autres types de relateurs comme «se composer de », «comprendre », «être constitué de », « réunir », qui construisent un tout dit « compositionnel », représenté « sous l'aspect d'une totalité formée à partir de l'ensemble d'éléments qui entrent dans sa composition ». (Tamba, 1994, 78) :

38) Le peuple français est constitué de français de souche française et de français de souches étrangères.

39) Paul fait partie du peuple français.

40) Le peuple gabonais est constitué de 50 ethnies issues de diverses régions. (J. Leclerc, Gabon, 2009, 2).

41) Le peuple est constitué de travailleurs, de prolétaires, qui à aucun moment ne profitent des fruits de leur labeur. (S. Peltier, Fourier: de l'industrie civilisée à une pathocratie associative).

Le résultat obtenu par ce type de relateurs offre des éléments très hétérogènes : des sous-groupes comme dans les exemples (38) et (40), des noms relatifs à des emplois particuliers; travailleurs et prolétaires. Nous ne pouvons donc pas parler de «méronyme » puisque ces emplois ne commutent pas entre eux de par leur emploi contextualisé.

Par ailleurs, comme l'indique Lammert : «les définitions lexicographiques des Ncolls nous renseignent sur la manière dont le lien s'établit entre éléments et collections. » (Lammert, 2010, 114). Ces définitions peuvent nous éclairer sur la composition de ces noms, et ce en mettant en avant les noms d'éléments. Dans le cas de peuple, les différentes définitions offrent des termes génériques (humain, individu) :

42) Ensemble des humains vivant en société sur un territoire déterminé. (TLFi)

43) Ensemble des individus constituant une nation. (TLFi). 
Les syntagmes binominaux constituent un autre procédé d'extraction d'éléments. Ils sont de deux types :

- «L'un reliant les éléments à leur collection, à savoir Dét Nelt de Dét Ncoll

- L'autre explicitant quels sont les éléments de la collection avec la structure Dét Ncoll de Nelt ». (Lammert, 2010, 115).

C'est le deuxième type qui nous intéresse dans le cas de peuple, car les structures comme : un homme du peuple, une femme du peuple, les gens du peuple relèvent d'un emploi particulier désignant «les personnes issues de la couche la plus démunie ». Selon le modèle Dét Ncoll de Nelt nous retrouvons dans le corpus des exemples tels que :

44) Le peuple d'artistes

45) Un peuple de travailleurs

Bien que renvoyant à des noms d'humains, la structure Dét Ncoll de Nelt se rapproche plus d'un emploi quantificateur.

\subsection{Peuple : un nom d'humain}

Peuple est un terme qui représente l'humain au collectif. Dans les définitions lexicographiques, le trait [+hum] apparaît d'emblée. Cependant en examinant son environnement syntagmatique (les verbes, les adjectifs, les compléments de noms, etc.), nous relevons dans le corpus des constructions qui associent peuple à l'être humain. Nous passons d'un ensemble d'individus à une unité ayants des attributs humains.
46) Les formes vraiment fixes ne sont pas fixes mais figées, donc sont virtuellement mortes (ce qui ne les empêche pas, contrairement à ce qu'un vain peuple avant-gardiste pense, de pouvoir, tels des phénix, de leurs cendres parfois renaître). (J. Roubaud, Récit 2000, p72)
47) C'est la femme en cheveux de la libération, celle que tant de peuples ont aimée, grande, blanche, rose et rouge, une voix argentine, toujours tendre, et un peu étonnée. (P. Guyotat, Formation, 2007, p 137-138)

Ainsi, il est employé, comme le montre le tableau $(2)^{18}$ ci-dessous avec les verbes psychologiques :

\begin{tabular}{|l|l|l|l|l|l|}
\hline Communication & Don/Privation & Entrée/Sortie & Frapper/Toucher & $\begin{array}{c}\text { Etats } \\
\text { physiques et } \\
\text { comportements }\end{array}$ & Locatif \\
\hline Caqueter & Sacrifier & Surgir & Vaincre & Agir & Siéger \\
Grommeler & Payer & Retourner & Résister & Vivre \\
& Subir & Descendre & balayer & Contrôler \\
& & S'éloigner & & S'organiser & \\
& & & & & \\
\hline
\end{tabular}




\begin{tabular}{|c|c|c|c|c|c|}
\hline $\begin{array}{l}\text { Mouvement } \\
\text { sur place }\end{array}$ & $\begin{array}{l}\text { Verbes } \\
\text { psychologiques }\end{array}$ & $\begin{array}{l}\text { Réalisation/ } \\
\text { mise en état }\end{array}$ & $\begin{array}{l}\text { Saisir/serrer/ } \\
\text { posséder }\end{array}$ & Union/Réunion & $\begin{array}{l}\text { Verbes } \\
\text { auxiliaires }\end{array}$ \\
\hline Se soulever & $\begin{array}{l}\text { Vouloir } \\
\text { Penser } \\
\text { S'éteindre } \\
\text { Se mêler } \\
\text { Connaître } \\
\text { Ignorer } \\
\text { Se lasser } \\
\text { Souffrir } \\
\text { Se douter } \\
\text { Persister } \\
\text { Aimer } \\
\text { Trouver } \\
\text { Soutenir } \\
\text { Accepter } \\
\text { Surveiller }\end{array}$ & Fonder & $\begin{array}{l}\text { Avoir } \\
\text { Trouver } \\
\text { Hanter } \\
\text { Posséder } \\
\text { Quitter } \\
\text { Prendre } \\
\text { Elire }\end{array}$ & $\begin{array}{l}\text { Se rallier } \\
\text { Se réconcilier } \\
\text { S'opposer }\end{array}$ & $\begin{array}{l}\text { Exister } \\
\text { Pouvoir } \\
\text { Disparaitre } \\
\text { Demeurer } \\
\text { devoir }\end{array}$ \\
\hline
\end{tabular}

Tableau 2. Verbes utilisés avec peuple en fonction sujet.

Il est également employé avec des adjectifs qui appellent normalement un sujet humain singulier :

48) Ah! Il est beau le peuple! Mais il ainsi parce qu'il est mal élevé, mal éduqué. (D. Bardet, Cahiers de jeunesse de Denise Bardet, institutrice à Oradour-sur-Glane, p 33-34).

49) Le peuple studieux siégeait sur l'aile gauche de la classe, la plus proche des fenêtres, juste sous le regard professoral. (A. Caretta, Pas un jour, 2002, p 119).

En fonction complément du nom, peuple entre dans des structures collocatives avec des noms de parties du corps ou des noms relatifs au corps humain :

50) Le peuple italien a été la première victime de Mussolini, et c'est de la main de ce peuple qu'il devra expier. (M. Duras, Cahiers de la guerre et autres textes, 2006, p 239-240).

51) Il n'ose pas parler des camps de concentration, il répugne manifestement à intégrer les pleurs du peuple dans la victoire. (M. Duras, Cahiers de la guerre et autres textes, 2006, p 213-214).

52) Les empires coloniaux, gros ou petits, emploient toujours les mêmes procédés pour farcir les oreilles de leurs peuples avec des mensonges. (J. Roubaud, Impératif catégorique, 2008, p 76-77).

Ces emplois qui rapprochent peuple de l'être humain, montrent que les éléments sont conceptualisés comme étants une seule personne masquant ainsi la pluralité sémantique de ce nom collectif. 


\section{Pour conclure}

Cette contribution a permis de recenser un certain nombre de problèmes relatifs à la classe des NCH et montre qu'elle ne peut être appréhendée de la même façon que les collectifs d'inanimés. Désignant des ensembles dans la totalité, peuple résiste au principe de la divisibilité et à celui de la référence cumulative et ne possède pas de méronyme à proprement parlé. Ses emplois dans $\$ 4.5$ le rapprochant d'un être humain montrent que la cohésion des groupes humains peut troubler leur pluralité sémantique. Cela nous invite à approfondir, d'une part, notre réflexion sur les modes de structuration et de formation des collectifs humains, et d'une autre part, d'affiner notre tentative de classement afin de construire des classes les plus homogènes possible.

Se pose également la question de ce que nous rangeons, par extension, dans la classe des NCH puisque les noms issus d'un même procédé métonymique ne donnent pas tous lieu à des collectifs humains :

53) La jeunesse s'est rassemblée en nombre ce jeudi 20 juillet. (Google)

54) *la vieillesse s'est rassemblée en nombre ce jeudi 20 juillet.

55) *l'enfance s'est rassemblée en nombre ce jeudi 20 juillet.

56) *l'adolescence s'est rassemblée en nombre ce jeudi 20 juillet.

Est-ce une simple question d'autorisation parentale pour les exemples (55), (56) ou bien une intéressante piste linguistique à explorer?

\section{Références bibliographiques}

Benninger, C. (1999). De la quantité aux substantifs quantificateurs, Paris : Klincksieck.

Borillo, A. (1997). Statut et mode d'interprétation des noms collectifs, in C. Guimier (éd.), Cotexte et calcul de sens, Caen, Presses Universitaires de Caen, 105-121.

Cruse, D.A. (1986). Lexical semantics, Cambridge : Cambridge University Press.

Cappeau, P et Schnedecker, C. (2014). Gens, personne(s), individu(s) : trois saisies de l'humain. F. Neveu, P.

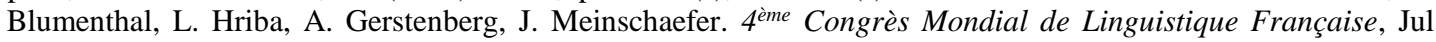
2014, Berlin, Germany. Volume 8, 2014 (8), 3027-3040. <Hal-01058191>.

Dubois, J et Dubois-Charlier, F. (1996). Collectifs d'êtres vivants. Linx, 34-35, 125-132.

Dubois, J et Dubois-Charlier, F. (2007). Présentation de la classification des verbes français de Jean Dubois et de Françoise Dubois-Charlier. Langue française, 53, 3-19.

Flaux, N. (1999). A propos des noms collectifs. Revue de linguistique romane, 63, 471-502.

Gréa, P. (2012). Etre une/Faire partie de : not a piece of cake, in Constructions in French, Bouveret, M. et Legallois, D. (éds), 73-97. Amsterdam : John Benjamins.

Gross, G. (2009). Sur le statut syntaxique des substantifs humains, in Mélanges offerts à Jean Claude Ancombre, Presses de l'Université de Savoie, 27-41.

Gross, G. (2011). Classification sémantique des collectifs humains. Cahiers de lexicologie, 98-1, 65-81.

Lammert, M. (2010). Sémantique et cognition : les noms collectifs, Genève : Droz.

Lammert, M. et Lecolle, M. (2014). Les noms collectifs en français, un état des lieux. Cahiers de Lexicologie, 105, 2014-2, 203-222.

Lecolle, M. (1998). Noms collectifs et méronymie. Cahiers de grammaire, 23, 41-65.

Lecolle, M. (2008). Identité/altérité et noms collectifs humains. Le cas de communauté. Questions de communication, 13, 323-342. 
Lecolle, M. (2013). Noms collectifs humains : un point de vue de sémantique lexicale sur l'identité dans le rapport individu/groupe. \& Interrogations ?, 16.

Lecolle, M. (2014). Des noms collectifs aux noms collectifs humains. Propriétés communes et spécificités. Communication présentée aux journées NHUMA 6, Strasbourg, France.

Mihatsch, W. (2015). Collectives, in Müller, P.O., Ohnheiser, I., Olsen, S., Rainer, F. (eds), HSK Word-Formation. An International Handbook of Languages of Europe. Berlin: Mouton De Gruyter.

Tamba, I. (1994). Un puzzle sémantique : le couplage de tout à partie et de partie à tout. Le gré des langues, 7, 64-85.

${ }^{1}$ Gross (2011), Lecolle (2008, 2013, 2014)

${ }^{2}$ Nous avons constitué une base de données regroupant un échantillon de NCH obtenu par une recherche inverse à partir des définitions. Nous avons imaginé les combinaisons possibles pour rendre compte du trait [+hum] et du trait [+collectif].

${ }^{3}$ Nous rassemblons sous cette étiquette le nom humanité que nous considérons comme le nom collectif humain général puisqu'il représente l'ensemble de tous les hommes, ainsi que des noms comme biotype.

${ }^{4}$ Lammert emploie le terme de méta-collectifs pour désigner les termes de groupe, ensemble et collection.

${ }^{5}$ Nous nous sommes posée la question de l'utilité des deux définisseurs groupe de personnes et groupe d'individus et nous avons remarqué qu'individu a été utilisé pour les définitions relevant de domaines bien particuliers, à savoir la sociologie pour le nom bande, et la démographie pour population.

${ }^{6}$ La notion d'espace est à prendre au sens large, puisqu'il existe aussi des regroupements virtuels organisés via des réseaux sociaux.

${ }^{7}$ Le client en effectuant son achat n'a pas l'intention d'appartenir à la clientèle du magasin.

${ }^{8}$ Base de données multilingues, université de Leipzig.

${ }^{9}$ Pour limiter les effets de sens particuliers.

${ }^{10}$ Ainsi que Google.

${ }^{11}$ Nous n'aurons pas de combinaison [+social] [+spatial] puisque l'appartenance à une classe sociale se fait en dehors de tout « espace » commun.

${ }^{12}$ Nous avons collecté ces tests dans Lammert, 2010.

${ }^{13}$ Il s'agit ici de l'acception du peuple Français en tant que 63379349 personnes (chiffre au $1^{\text {er }}$ janvier 2013).

${ }^{14}$ Cela est valable jusqu'à une certaine limite puisque cet emploi est hyperbolique et suppose un très grand nombre de personnes.

15 Termes empruntés à Lammert $(2010,102)$.

${ }^{16}$ Dans le cadre de ce travail nous nous sommes intéréssée aux seuls emplois nominaux de peuple, toutefois, il peut avoir le fonctionnement d'un substantif quantificateur. Nous relevons dans le corpus soit les structures, un peuple de, tout un peuple de, soit la structure massive du peuple. Dans les deux cas l'accent est mis sur la quantité, nous perdons le sens dénotatif d'un ensemble d'humains.

${ }^{17}$ Des noms comme foule, ethnie, famille, etc.

${ }^{18}$ Cette répartition a été construite selon les 14 classes génériques de la classification des verbes en français de J. Dubois et F. Dubois-Charlier. 